\title{
A new approach to the existence of quasiperiodic solutions for a class of semilinear Duffing-type equations with time-periodic parameters
}

Xiaoming Wang ${ }^{1,2^{*}}$, Lixia Wang ${ }^{3}$ and Hainv Tan ${ }^{1}$

"Correspondence:
wxmsuda03@163.com
1School of Mathematics \&
Computer Science, Shangrao
Normal University, Shangrao, Jiangxi
334001, China
${ }^{2}$ Chern Institute of Mathematics,
Nankai University, Tianjin, 300071,
China
Full list of author information is
available at the end of the article

available at the end of the article

\begin{abstract}
Let $q(t)$ be a continuous $2 \pi$-periodic function with $\frac{1}{2 \pi} \int_{0}^{2 \pi} q(t) d t>0$. We propose a new approach to establish the existence of Aubry-Mather sets and quasi-periodic solutions for the following time-periodic parameters semilinear Duffing-type equation:

$$
x^{\prime \prime}+q(t) x+f(t, x)=0
$$

where $f(t, x)$ is a continuous function, $2 \pi$-periodic in the first argument and continuously differentiable in the second one. Under some assumptions on the functions $q$ and $f$, we prove that there are infinitely many generalized quasi-periodic solutions via a version of the Aubry-Mather theorem given by Pei. Especially, an advantage of our approach is that it does not require any high smoothness assumptions on the functions $q(t)$ and $f(t, x)$.
\end{abstract}

MSC: $34 \mathrm{C} 12 ; 37 \mathrm{C} 55$

Keywords: Duffing-type equation; semilinear; Aubry-Mather sets; quasi-periodic solutions

\section{Introduction}

The goal of this paper is to study the existence of Aubry-Mather sets and quasi-periodic solutions to the following time-periodic parameters semilinear Duffing-type equation:

$$
x^{\prime \prime}+q(t) x+f(t, x)=0,
$$

where $q(t)$ is continuous and $2 \pi$-periodic function in the time $t, f(t, x)$ is a continuous function, $2 \pi$-periodic in the first argument and continuously differentiable in the second one. As is well known such a system is one of the most important models in Hamiltonian systems due to both its physical significance and mathematical fascination despite its simple form. Here we are concerned with the so-called break-down of stability (in the sense of the Lagrangian) by using the Aubry-Mather theory.

(c) 2016 Wang et al. This article is distributed under the terms of the Creative Commons Attribution 4.0 International License (http://creativecommons.org/licenses/by/4.0/), which permits unrestricted use, distribution, and reproduction in any medium, provided you give appropriate credit to the original author(s) and the source, provide a link to the Creative Commons license, and indicate if changes were made. 
In the early 1980s, Aubry [1] and Mather [2] proved independently that invariant curves of integrable system will be broken if its perturbation increased gradually and/or the smoothness of integrable system is weakened, when they, respectively, studied onedimensional liquid crystal model of solid state physics and the qualitative properties of the orbits of an area-preserving twist map of the annulus. They also found that when invariant curves break, they do not simply disappear, some special invariant sets still exist. Today, these sets are called Aubry-Mather sets. For the planar differential system, AubryMather theory suggests that its Poincaré mapping has Aubry-Mather sets $M_{\sigma}$ with a rotation number $\sigma$, then the planar differential system possesses Aubry-Mather type solutions $z_{\sigma}(t)=\left(x_{\sigma}(t), y_{\sigma}(t)\right)$, such that $M_{\sigma} \equiv \overline{\left\{z_{\sigma}(2 \pi i), i \in Z\right\}}$ with the following geometrical and dynamical properties:

(1) if $\sigma=\frac{n}{m} \in \mathbb{Q}$ with $(n, m)=1$, then $z_{\sigma}(t)$ is a Birkhoff periodic solution with periodic $2 m \pi$ and $\arg \left(z_{\sigma}(t)+m\right)=\arg \left(z_{\sigma}(t)\right)+n$, the $m$ solutions $z_{\sigma}(t+2 \pi i)(0 \leq i \leq m-1)$ can be homotopically drawn to $m$ straight lines;

(2) if $\sigma \in \mathbb{R} \backslash \mathbb{Q}$, then $M_{\sigma}$ is either an invariant circle and its orbits are just usual quasi-periodic orbits, or an invariant Cantor set and its orbits become generalized ones.

In general, we note that the existence of Birkhoff type periodic solution is very difficult to prove, see, for example, Bernstein and Katok's work [3]. But Aubry-Mather theory has provided a powerful tool for the in-depth study of the dynamic behavior of differential equations. Since the pioneering work of Aubry [1] and Mather [2], Aubry-Mather sets for area-preserving monotone twist homeomorphism have been widely studied due to their applications in many fields such as one-dimensional crystal model of solid state physics, differential geometry and dynamical systems (see $[4,5])$. And then much work has been carried out concerning the existence of Aubry-Mather sets for various kinds of differential equations, such as Hamiltonian systems [6-9], reversible systems [10, 11] and nonlinear asymmetric oscillator [12-15].

In the 1990s, the Duffing-type equation has been a typical model in the recent AubryMather theory for planar periodic Hamiltonian systems, there are several papers concerning this problem for the Duffing equation (see [16-18]). For example, Pei [16] and Qian [17] have proved, respectively, the existence of Aubry-Mather sets and quasi-periodic solutions for some superlinear Duffing equations and sublinear Duffing equations by using Aubry-Mather theory under some suitable assumptions.

When $q(t)=\lambda^{2}$ is constant and $f(x, t)=\varphi(x)-p(t)$, equation (1.1) reduces to the semilinear Duffing equation

$$
x^{\prime \prime}+\lambda^{2} x+\varphi(x)=p(t)
$$

In 1994, based on a generalized version of Aubry-Mather theorem, Pei [18] obtained the existence of Aubry-Mather sets of equation (1.2) for any continuous $2 \pi$-periodic function $p(t)$ if

(A) $\frac{\varphi(x)}{x}>\varphi^{\prime}(x)>0$, for $|x| \geq d>0$;

( $\left.\mathrm{A}_{2}\right) \varphi(x)=o(x),\left|\varphi(x)-x \varphi^{\prime}(x)\right| \rightarrow+\infty$, as $|x| \rightarrow+\infty ;\left|x \varphi^{\prime \prime}(x)\right| \leq C$, where the constant $C>0$.

An interesting question is: can the smoothness requirement of the perturbation term $\varphi(x) \in C^{2}(\mathbb{R})$ for equation (1.2) be weakened? 
Recently, the author [19] has extended such a result to the case $\varphi(x) \in C^{1}(\mathbb{R})$ and obtained the existence of Aubry-Mather sets and quasi-periodic solutions for equation (1.2) under the following conditions:

(B $\left.\operatorname{B}_{1}\right) \lim _{|x| \rightarrow+\infty} \varphi^{\prime}(x)=0$;

$\left(\mathrm{B}_{2}\right) \operatorname{sgn}(x)\left(\varphi(x)-x \varphi^{\prime}(x)\right)>2 p_{\infty}$, for $|x| \geq d>0$, where $p_{\infty}=\max _{t \in[0,2 \pi]}|p(t)|$.

In this paper, we will continue the study of the existence of Aubry-Mather sets and quasiperiodic solutions of equation (1.1) initiated in $[18,19]$. In our case, we cannot apply the estimation method used in [18]. Instead, we seek a suitable action and angle variable transformation so that the transformed system of (1.1) is a perturbation of an integral Hamiltonian system, and then propose a new estimate approach and apply a kind of analytical techniques developed by the present author (see the recent papers $[11,14,15]$ ) to directly prove the Poincaré map of the transformed system satisfying monotone twist property, which leads to our desired results. Especially, an advantage of our approach is that it does not require any high smoothness assumptions on the functions $q(t)$ and $f(t, x)$. The results obtained in this paper are natural generalizations and refinements of the results obtained in $[18,19]$.

Our main result is the following.

Theorem 1.1 Assume that equation (1.1) satisfies:

$\left(\mathrm{D}_{1}\right) q(t) \in C\left(\mathbf{S}^{1}\right)$ and $\frac{1}{2 \pi} \int_{0}^{2 \pi} q(t) d t=\gamma^{2}>2 b_{\infty}$, where $\gamma>0$,

$$
b_{\infty}=\max _{t \in[0,2 \pi]}|b(t)|, \quad b(t)=q(t)-\gamma^{2}
$$

$\left(\mathrm{D}_{2}\right) f(t, x) \in C^{0,1}\left(\mathbf{S}^{1} \times \mathbb{R}\right)$ and has limits

$$
\lim _{|x| \rightarrow+\infty} f_{x}(t, x)=0, \quad \text { uniformly in } t \in[0,2 \pi]
$$

$\left(D_{3}\right)$ there is a constant $d>0$, such that

$$
\operatorname{sgn}(x)\left[f(t, x)-x f_{x}(t, x)\right]>2 b_{\infty}, \quad \text { for }|x| \geq d
$$

Then there exists $\varepsilon_{0}>0$, such that for any $\alpha \in\left(2 \gamma \pi, 2 \gamma \pi+\varepsilon_{0}\right)$, equation (1.1) possesses an Aubry-Mather type solution $z_{\alpha}(t)=\left(x_{\alpha}(t), x_{\alpha}^{\prime}(t)\right)$ with rotation number $\alpha$, that is:

(i) if $\alpha=\frac{n}{m}$ is rational, and $(n, m)=1$, the solution $z_{\alpha}^{i}(t)=z_{\alpha}(t+2 \pi i), 0 \leq i \leq m-1$, are mutually unlinked periodic solutions of period $m$;

(ii) if $\alpha$ is irrational, the solution $z_{\alpha}(t)$ either a usual quasi-periodic solution or a bounded solution such that the closed set

$$
M_{\alpha} \equiv \overline{\left\{z_{\alpha}(2 \pi i), i \in \mathbb{Z}\right\}}
$$

is a Denjoy minimal set (see the definition of it in [20]).

Remark 1.1 Applying the rule of L'Hospital to condition $\left(D_{2}\right)$, it is easy to see that 
$\left(f_{0}\right)$

$$
\lim _{|x| \rightarrow+\infty} \frac{f(t, x)}{x}=0, \quad \text { uniformly in } t \in[0,2 \pi]
$$

Example 1.1 Let $q(t)=1+\frac{1}{4} \sin t$ and $f(t, x)=\operatorname{sgn}(x) \ln (1+|x|) \cdot(1+|p(t)|)$, where $p(t)$ is a continuous function with $p(t+2 \pi)=p(t)$. Then $q(t) \in C\left(\mathbf{S}^{1}\right)$ and $f(t, x)$ meet the conditions of $\left(D_{1}\right)-\left(D_{3}\right)$ in Theorem 1.1. We can check it as follows: (i) By simple calculation, we have $\gamma=1, b_{\infty}=\max _{t \in[0,2 \pi]}\left|\frac{1}{4} \sin t\right|=\frac{1}{4}$, then $1=\gamma^{2}>2 b_{\infty}=\frac{1}{2}$; (ii) since $f_{x}(t, 0)=1+|p(t)|$ and $f_{x x}(t, 0)=\infty$, we have $f(t, x) \in C^{0,1}\left(\mathbf{S}^{1} \times \mathbb{R}\right)$ and it is obvious that

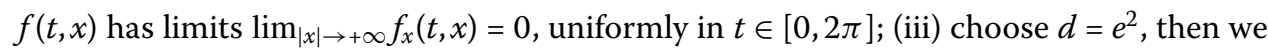
have $\operatorname{sgn}(x)\left[f(t, x)-x f_{x}(t, x)\right] \geq 1>\frac{1}{2}=2 b_{\infty}$, for $|x| \geq d$. Thus, according to (i)-(iii), the assumptions $\left(\mathrm{D}_{1}\right)-\left(\mathrm{D}_{3}\right)$ in Theorem 1.1 hold.

Remark 1.2 It is easy to verify that the results in $[18,19]$ cannot be applied to Example 1.1 to obtain the existence of Aubry-Mather sets and quasi-periodic solutions. Therefore, the results obtained in this paper can be viewed as natural generalizations and refinements of the results in $[18,19]$.

Remark 1.3 It seems that the break-down of stability (in the sense of the Lagrangian) is related to the smoothness of $f(t, x)$. And we do not know whether or $\operatorname{not} f(t, x) \in C^{0,0}\left(\mathbf{S}^{1} \times \mathbb{R}\right)$ is sufficient to guarantee the existence of Aubry-Mather sets and quasi-periodic solutions of equation (1.1).

The main idea of our proof is acquired from [21]. The proof of Theorem 1.1 is based on a version of Aubry-Mather theorem due to Pei [18]. The rest of this paper is organized as follows. In Section 2, we introduce the action-angle variables which transform equation (1.1) into a perturbation of an integral Hamiltonian system. In Section 3, we will show that the Poincaré map of the equivalent system satisfies the monotone twist property around infinity, then some results can be obtained.

\section{Action-angle variables and some properties}

Let $q(t)=\gamma^{2}+b(t)$. Then $b(t)$ is a $2 \pi$ periodic function and $\frac{1}{2 \pi} \int_{0}^{2 \pi} b(t) d t=0$. Hence, equation (1.1) is equivalent to the system

$$
\dot{x}=-y, \quad \dot{y}=\left(\gamma^{2}+b(t)\right) x+f(t, x) .
$$

Now we introduce the action and angle variables $(I, \theta)$ as follows:

$$
x=\sqrt{\frac{2 I}{\gamma}} \cos \theta, \quad y=\sqrt{2 \gamma I} \sin \theta,
$$

where $I>0$ and $\theta \in \mathbf{S}^{1}=\mathbb{R} / 2 \pi \mathbb{Z}$, then it is not difficult to prove that the mapping $\Psi: \mathbf{S}^{1} \times(0, \infty) \rightarrow \mathbb{R}^{2}-\{0\},(\theta, I) \mapsto(x, y)$ is a canonical transformation, such that (2.1) is transformed into

$$
\dot{\theta}=\Phi_{1}(t, \theta, I), \quad \dot{I}=\Phi_{2}(t, \theta, I),
$$


where

$$
\begin{aligned}
& \Phi_{1}(t, \theta, I)=\gamma+\frac{x(\theta, I)(b(t) x(\theta, I)+f(t, x(\theta, I)))}{2 I}, \\
& \Phi_{2}(t, \theta, I)=\frac{y(\theta, I)(b(t) x(\theta, I)+f(t, x(\theta, I)))}{\gamma} .
\end{aligned}
$$

Under the assumptions of $\left(D_{2}\right)$ and $\left(D_{3}\right)$, it is easy to prove the existence and uniqueness of the solution of the initial value problem associated with (2.3). Moreover, this solution has continuous derivatives with respect to initial data.

Let $\left(\theta\left(t ; \theta_{0}, I_{0}\right), I\left(t ; \theta_{0}, I_{0}\right)\right)$ be the solution of $(2.3)$ with initial value $\theta(0)=\theta_{0}$ and $I(0)=I_{0}$. Then $x\left(t ; \theta_{0}, I_{0}\right)=x\left(\theta\left(t ; \theta_{0}, I_{0}\right), I\left(t ; \theta_{0}, I_{0}\right)\right)=\sqrt{\frac{2 I\left(t ; \theta_{0}, I_{0}\right)}{\gamma}} \cos \theta\left(t ; \theta_{0}, I_{0}\right)$ is the solution of $(1.1)$.

First, we give the following growth estimates as regards $I\left(t ; \theta_{0}, I_{0}\right)$ and $\theta\left(t ; \theta_{0}, I_{0}\right)$.

For the sake of convenience and simplicity, in the following, we let $\theta=\theta\left(t ; \theta_{0}, I_{0}\right), I=$ $I\left(t ; \theta_{0}, I_{0}\right)$ and $x=x(\theta, I)=x\left(\theta\left(t ; \theta_{0}, I_{0}\right), I\left(t ; \theta_{0}, I_{0}\right)\right), y=y(\theta, I)=y\left(\theta\left(t ; \theta_{0}, I_{0}\right), I\left(t ; \theta_{0}, I_{0}\right)\right)$.

\section{Lemma 2.1 The limit}

$$
\lim _{I_{0} \rightarrow+\infty} I\left(t ; \theta_{0}, I_{0}\right)=+\infty
$$

holds uniformly on $t \in[0,2 \pi]$.

Proof From $\left(\mathrm{f}_{0}\right)$ and coordinate transformation (2.2), there exist constants $C>0, K>0$, such that $\left|I^{\prime}(t)\right|=\left|\frac{y(b(t) x+f(t, x))}{\gamma}\right| \leq C I(t)+K, \forall I \neq 0$.

Then, by the Gronwall inequality, one has

$$
e^{-2 \pi C} I_{0}-\frac{K}{C}\left(1-e^{-2 \pi C}\right) \leq I(t) \leq e^{2 \pi C} I_{0}+\frac{K}{C}\left(e^{2 \pi C}-1\right)
$$

for all $t \in[0,2 \pi]$.

So, by (2.4), $I\left(t ; \theta_{0}, I_{0}\right) \rightarrow+\infty$ as $I_{0} \rightarrow+\infty$ uniformly for $t \in[0,2 \pi]$.

Lemma 2.2 There exist constants $k_{2}>k_{1}>0$ and $\bar{I}>0$, such that for any $I_{0} \geq \bar{I}$, we have:

(i) $\frac{\gamma}{2} \leq \theta^{\prime}\left(t ; \theta_{0}, I_{0}\right) \leq 2 \gamma, \forall \theta_{0} \in \mathbb{R}$ and $\forall t \in[0,2 \pi]$;

(ii) $k_{1} I_{0} \leq I\left(t ; \theta_{0}, I_{0}\right) \leq k_{2} I_{0}, \forall \theta_{0} \in \mathbb{R}$ and $\forall t \in[0,2 \pi]$.

Proof (i) Since $\left(\mathrm{f}_{0}\right)$ holds, for every $\varepsilon>0$ (we may restrict $0<\varepsilon<\frac{\gamma^{2}}{2}-b_{\infty}$ ), there exists $M=M(\varepsilon)>0$, such that

$$
|f(t, x)| \leq \varepsilon|x|
$$

if $|x| \geq M$ and $\forall t \in[0,2 \pi]$. Hence, by (2.3), we have

$$
\begin{aligned}
\frac{d \theta}{d t} & =\gamma+\frac{x(b(t) x+f(t, x))}{2 I} \geq \gamma-\frac{\left(b_{\infty}+\varepsilon\right) x^{2}}{2 I} \\
& \geq \gamma-\frac{b_{\infty}+\varepsilon}{\gamma} .
\end{aligned}
$$


By $\left(D_{1}\right)$, we have $\gamma^{2}>2 b_{\infty}$. Thus, we have

$$
\frac{d \theta}{d t} \geq \gamma-\frac{b_{\infty}+\varepsilon}{\gamma} \geq \frac{\gamma}{2}
$$

In the case $|x| \leq M$, we may assume that $|f(t, x)| \leq f_{\infty}$, where $f_{\infty}=\max \{|f(t, x)|: t \in$ $[0,2 \pi],|x| \leq M\}$, then by (2.2), we have

$$
\begin{aligned}
\frac{d \theta}{d t} & =\gamma+\frac{x(b(t) x+f(t, x))}{2 I} \geq \gamma-\frac{b_{\infty} x^{2}+f_{\infty}|x|}{2 I} \\
& \geq \gamma-\frac{b_{\infty}}{\gamma}-\frac{f_{\infty}}{\sqrt{2 \gamma I}} .
\end{aligned}
$$

So, by $\left(\mathrm{D}_{1}\right)$ and Lemma 2.1, there exists a constant $\bar{I}>0$, such that $\frac{d \theta}{d t} \geq \frac{\gamma}{2}$ if $I_{0} \geq \bar{I}$.

Similarly, the same argument as above shows that the inequality on the right side of (i) holds.

(ii) By (2.4), we can easily find constants $k_{2}>k_{1}>0$ and $\bar{I}>0$, such that

$$
k_{1} I_{0} \leq I(t) \leq k_{2} I_{0}
$$

for any $I_{0} \geq \bar{I}$ and $\forall t \in[0,2 \pi]$.

\section{Monotone twist property and proof of Theorem 1.1}

Let the Poincaré map $P$ of equation (2.3) be

$$
P:\left(\theta_{0}, I_{0}\right) \mapsto\left(\theta\left(2 \pi, \theta_{0}, I_{0}\right), I\left(2 \pi, \theta_{0}, I_{0}\right)\right) .
$$

To complete the proof of Theorem 1.1, one can see that it suffices to show that the Poincaré map $P$ is a monotone twist map around infinity, that is, we only need to prove $\frac{\partial \theta\left(2 \pi ; \theta_{0}, I_{0}\right)}{\partial I_{0}}<0$ if $I_{0} \gg 1$. Then the existence of Aubry-Mather sets and quasi-periodic solutions is guaranteed by a generalized Aubry-Mather theorem given by Pei [18].

In the following, we will investigate the behavior of $\frac{\partial \theta\left(2 \pi ; \theta_{0}, I_{0}\right)}{\partial I_{0}}$ when $I_{0} \gg 1$ by some lemmas. For the sake of convenience, in later discussions we also write $x, y, \theta, I$ instead of $x\left(\theta\left(t ; \theta_{0}, I_{0}\right), I\left(t ; \theta_{0}, I_{0}\right)\right), y\left(\theta\left(t ; \theta_{0}, I_{0}\right), I\left(t ; \theta_{0}, I_{0}\right)\right), \theta\left(t ; \theta_{0}, I_{0}\right), I\left(t ; \theta_{0}, I_{0}\right)$, respectively.

Lemma 3.1 The following convergences hold uniformly on $t \in[0,2 \pi]$ :

(i) $\frac{x f(t, x)}{I} \rightarrow 0, \frac{x^{2} f_{x}(t, x)}{I} \rightarrow 0$, as $I_{0} \rightarrow+\infty$;

(ii) $\frac{y f(t, x)}{I} \rightarrow 0, \frac{y x f_{x}(t, x)}{I} \rightarrow 0$, as $I_{0} \rightarrow+\infty$;

(iii) $\frac{f^{2}(t, x)}{I} \rightarrow 0, \frac{x f(t, x) f_{x}(t, x)}{I} \rightarrow 0, \frac{x^{2} f_{x}^{2}(t, x)}{I} \rightarrow 0$, as $I_{0} \rightarrow+\infty$.

Proof If $\left(\mathrm{D}_{2}\right)$ and $\left(\mathrm{f}_{0}\right)$ hold, then to each $\varepsilon>0$ there corresponds a positive number $M=$ $M(\varepsilon)>0$, if $|x| \geq M$ and $\forall t \in[0,2 \pi]$, and we have

$$
\left|f_{x}(t, x)\right| \leq \frac{\gamma}{4} \varepsilon
$$

and

$$
|f(t, x)| \leq \frac{\gamma}{4} \varepsilon|x|
$$


Denote $K_{1}(\varepsilon)=\max \{|f(t, x)|: t \in[0,2 \pi],|x| \leq M\}, K_{2}(\varepsilon)=\max \left\{\left|f_{x}(t, x)\right|: t \in[0,2 \pi]\right.$, $|x| \leq M\}$.

(i) According to the coordinate transformation (2.2), one has

$$
\begin{aligned}
& \left|\frac{x f(t, x)}{I}\right| \leq \frac{M K_{1}(\varepsilon)}{I}+\frac{\gamma \varepsilon x^{2}}{4 I} \leq \frac{M K_{1}(\varepsilon)}{I}+\frac{\varepsilon}{2} \\
& \left|\frac{x^{2} f_{x}(t, x)}{I}\right| \leq \frac{M^{2} K_{2}(\varepsilon)}{I}+\frac{\gamma \varepsilon x^{2}}{4 I} \leq \frac{M^{2} K_{2}(\varepsilon)}{r}+\frac{\varepsilon}{2} .
\end{aligned}
$$

Then, given $\bar{I}>0$, choose $I_{0}$ so that $I_{0} \geq \bar{I}$, by using Lemma 2.2(ii), provided

$$
I(t) \geq \max \left\{\frac{2 M K_{1}(\varepsilon)}{\varepsilon} ; \frac{2 M^{2} K_{2}(\varepsilon)}{\varepsilon} ; 1\right\},
$$

we have

$$
\left|\frac{x f(t, x)}{I}\right| \leq \varepsilon, \quad\left|\frac{x^{2} f_{x}(t, x)}{I}\right| \leq \varepsilon .
$$

Since $\varepsilon>0$ is arbitrary, the proof of (i) is complete.

(ii) Corresponding to (2.2), one can see that

$$
\begin{aligned}
& \left|\frac{y f(t, x)}{I}\right| \leq \frac{K_{1}(\varepsilon)|y|}{I}+\frac{\gamma \varepsilon|y||x|}{4 I} \leq \frac{\sqrt{2 \gamma} K_{1}(\varepsilon)}{\sqrt{I}}+\frac{\gamma \varepsilon}{2} \\
& \left|\frac{y x f_{x}(t, x)}{I}\right| \leq \frac{M|y| K_{2}(\varepsilon)}{I}+\frac{\gamma \varepsilon|y||x|}{4 I} \leq \frac{\sqrt{2 \gamma} K_{2}(\varepsilon)}{\sqrt{I}}+\frac{\gamma \varepsilon}{2} .
\end{aligned}
$$

Then, given $\bar{I}>0$, choose $I_{0}$ so that $I_{0} \geq \bar{I}$, by using Lemma 2.2(ii), provided

$$
I(t) \geq \max \left\{\frac{8 \gamma K_{1}^{2}(\varepsilon)}{\varepsilon^{2}} ; \frac{8 \gamma K_{2}^{2}(\varepsilon)}{\varepsilon^{2}} ; 1\right\}
$$

we have

$$
\left|\frac{y f(t, x)}{I}\right| \leq \frac{\varepsilon}{2}+\frac{\gamma \varepsilon}{2} ; \quad\left|\frac{y x f_{x}(t, x)}{I}\right| \leq \frac{\varepsilon}{2}+\frac{\gamma \varepsilon}{2} .
$$

Since $\varepsilon>0$ is arbitrary, (ii) is proved.

(iii) From (2.2) we deduce that

$$
\begin{aligned}
& \left|\frac{f^{2}(t, x)}{I}\right| \leq \frac{K_{1}^{2}(\varepsilon)}{I}+\frac{\gamma^{2} \varepsilon^{2}|x|^{2}}{16 I} \leq \frac{K_{1}^{2}(\varepsilon)}{I}+\frac{\gamma \varepsilon^{2}}{8} \\
& \left|\frac{x^{2} f_{x}^{2}(t, x)}{I}\right| \leq \frac{M^{2} K_{2}^{2}(\varepsilon)}{I}+\frac{\gamma^{2} \varepsilon^{2}|x|^{2}}{16 I} \leq \frac{M^{2} K_{2}^{2}(\varepsilon)}{I}+\frac{\gamma \varepsilon^{2}}{8} ; \\
& \left|\frac{x f(t, x) f_{x}(t, x)}{I}\right| \leq \frac{M K_{1}(\varepsilon) K_{2}(\varepsilon)}{I}+\frac{\gamma^{2} \varepsilon^{2}|x|^{2}}{16 I} \leq \frac{M K_{1}(\varepsilon) K_{2}(\varepsilon)}{I}+\frac{\gamma \varepsilon^{2}}{8} .
\end{aligned}
$$

Then, given $\bar{I}>0$, choose $I_{0}$ so that $I_{0} \geq \bar{I}$, by using Lemma 2.2(ii), provided

$$
I(t) \geq \max \left\{\frac{8 K_{1}^{2}(\varepsilon)}{\varepsilon} ; \frac{8 M^{2} K_{2}^{2}(\varepsilon)}{\varepsilon} ; \frac{8 M K_{1}(\varepsilon) K_{2}(\varepsilon)}{\varepsilon} ; 1\right\},
$$


we have

$$
\left|\frac{f^{2}(t, x)}{I}\right| \leq \frac{\varepsilon}{8}+\frac{\gamma \varepsilon^{2}}{8} ; \quad\left|\frac{x^{2} f_{x}^{2}(t, x)}{I}\right| \leq \frac{\varepsilon}{8}+\frac{\gamma \varepsilon^{2}}{8} ; \quad\left|\frac{x^{2} f_{x}^{2}(t, x)}{I}\right| \leq \frac{\varepsilon}{8}+\frac{\gamma \varepsilon^{2}}{8} .
$$

Since $\varepsilon>0$ is arbitrary, we get (iii).

For $t \in[0,2 \pi]$, set

$$
\begin{aligned}
& a_{1}(t)=\frac{\partial \Phi_{1}(t, \theta, I)}{\partial I}=\frac{-x\left[f(t, x)-x f_{x}(t, x)\right]}{4 I^{2}}, \\
& a_{2}(t)=\frac{\partial \Phi_{1}(t, \theta, I)}{\partial \theta}=a_{21}(t)+a_{22}(t), \quad \text { where } \\
& a_{21}(t)=\frac{-y\left(f(t, x)+x f_{x}(t, x)\right)}{2 \gamma I}, \quad a_{22}(t)=\frac{-b(t) y x}{2 \gamma I} ; \\
& a_{3}(t)=\frac{\partial \Phi_{2}(t, \theta, I)}{\partial \theta}=x[b(t) x+f(t, x)]-\frac{y^{2}\left[b(t)+f_{x}(t, x)\right]}{\gamma^{2}} .
\end{aligned}
$$

Using Lemma 2.1, Lemma 2.2, and Lemma 3.1, we have the following.

Lemma 3.2 For $t, s \in[0,2 \pi]$, the following conclusions hold:

(i) $a_{1}(t)=o\left(\frac{1}{I_{0}}\right)$, as $I_{0} \rightarrow+\infty$;

(ii) $a_{21}(t)=o(1) ;\left|a_{22}(t)\right| \leq \frac{b_{\infty}}{\gamma}$ as $I_{0} \rightarrow+\infty$;

(iii) $a_{1}(t) \cdot a_{3}(s)=o(1)$, as $I_{0} \rightarrow+\infty$.

Here and below o(1) denotes a generic infinitesimal as $I_{0} \rightarrow \infty$.

Let us consider the variational equation (2.3) with respect to the initial value $I_{0}$. One can verify that

$$
\dot{\theta}_{I_{0}}=a_{1}(t) \frac{\partial I}{\partial I_{0}}+a_{2}(t) \frac{\partial \theta}{\partial I_{0}}, \quad \dot{I}_{I_{0}}=-a_{2}(t) \frac{\partial I}{\partial I_{0}}+a_{3}(t) \frac{\partial \theta}{\partial I_{0}} .
$$

For convenience, we set $\sigma_{1}=e^{\frac{-2 \pi b_{\infty}}{\gamma}}, \sigma_{2}=e^{\frac{2 \pi b_{\infty}}{\gamma}}$. Then we have the following.

Lemma 3.3 For all $t \in(0,2 \pi], I_{0} \rightarrow+\infty$, we have:

(i) $\theta_{I_{0}}\left(t ; \theta_{0}, I_{0}\right) \rightarrow 0$;

(ii) $\sigma_{1}(1+o(1)) \leq I_{I_{0}}\left(t ; \theta_{0}, I_{0}\right) \leq \sigma_{2}(1+o(1))$;

(iii) $\sigma_{1}(1+o(1)) \leq \theta_{\theta_{0}}\left(t ; \theta_{0}, I_{0}\right) \leq \sigma_{2}(1+o(1))$.

Proof From the variational equations (3.1), one has

$$
\begin{aligned}
\theta_{I_{0}}(t) & =e^{\int_{0}^{t} a_{2}(s) d s} \cdot \int_{0}^{t} e^{-\int_{0}^{s} a_{2}(t) d t} a_{1}(s) \cdot I_{I_{0}}(s) d s \\
& =e^{\int_{0}^{t}\left(a_{21}(s)+a_{22}(s)\right) d s} \cdot \int_{0}^{t} e^{-\int_{0}^{s}\left(a_{21}(t)+a_{22}(t)\right) d t} a_{1}(s) \cdot I_{I_{0}}(s) d s,
\end{aligned}
$$

here we have used $\theta_{I_{0}}(0)=0$. Then, if $\int_{0}^{t} a_{1}(s) \cdot I_{I_{0}}(s) d s \geq 0$ for $t \in(0,2 \pi]$, by Lemma 3.2(ii), we have

$$
\sigma_{1}^{2}(1+o(1)) \int_{0}^{t} a_{1}(s) \cdot I_{I_{0}}(s) d s \leq \theta_{I_{0}}(t) \leq \sigma_{2}^{2}(1+o(1)) \int_{0}^{t} a_{1}(s) \cdot I_{I_{0}}(s) d s
$$


and if $\int_{0}^{t} a_{1}(s) \cdot I_{I_{0}}(s) d s \leq 0$ for $t \in(0,2 \pi]$, by Lemma 3.2(ii), we have

$$
\sigma_{1}^{2}(1+o(1)) \int_{0}^{t} a_{1}(s) \cdot I_{I_{0}}(s) d s \geq \theta_{I_{0}}(t) \geq \sigma_{2}^{2}(1+o(1)) \int_{0}^{t} a_{1}(s) \cdot I_{I_{0}}(s) d s .
$$

On the other hand, by the variational equations (3.1), we have

$$
\begin{aligned}
I_{I_{0}}(t) & =e^{-\int_{0}^{t} a_{2}(s) d s} \cdot\left(1+\int_{0}^{t} e^{\int_{0}^{s} a_{2}(t) d t} a_{3}(s) \cdot \theta_{I_{0}}(s) d s\right) \\
& =e^{-\int_{0}^{t}\left(a_{21}(s)+a_{22}(s)\right) d s} \cdot\left(1+\int_{0}^{t} e^{\int_{0}^{s}\left(a_{21}(t)+a_{22}(t)\right) d t} a_{3}(s) \cdot \theta_{I_{0}}(s) d s\right),
\end{aligned}
$$

here we have used $I_{I_{0}}(0)=1$. Now we will discuss it in the following two cases:

Case 1. If $\int_{0}^{t} a_{1}(s) \cdot I_{I_{0}}(s) d s \geq 0$ and $a_{3}(t) \geq 0$ for $t \in(0,2 \pi]$, or $\int_{0}^{t} a_{1}(s) \cdot I_{I_{0}}(s) d s \leq 0$ and $a_{3}(t) \leq 0$ for $t \in(0,2 \pi]$, then by Lemma 3.2(ii) and (iii), we have

$$
\begin{aligned}
I_{I_{0}}(t) & \leq \sigma_{2}(1+o(1))+\sigma_{2}^{4}(1+o(1)) \int_{0}^{t} a_{3}(s) \cdot\left(\int_{0}^{s} a_{1}(t) \cdot I_{I_{0}}(t) d t\right) d s \\
& =\sigma_{2}(1+o(1))+o(1) \int_{0}^{t} \int_{0}^{s} I_{I_{0}}(t) d t d s
\end{aligned}
$$

and

$$
\begin{aligned}
I_{I_{0}}(t) & \geq \sigma_{1}(1+o(1))+\sigma_{1}^{4}(1+o(1)) \int_{0}^{t} a_{3}(s) \cdot\left(\int_{0}^{s} a_{1}(t) \cdot I_{I_{0}}(t) d t\right) d s \\
& =\sigma_{1}(1+o(1))+o(1) \int_{0}^{t} \int_{0}^{s} I_{I_{0}}(t) d t d s .
\end{aligned}
$$

Case 2. If $\int_{0}^{t} a_{1}(s) \cdot I_{I_{0}}(s) d s \geq 0$ and $a_{3}(t) \leq 0$ for $t \in(0,2 \pi]$, or $\int_{0}^{t} a_{1}(s) \cdot I_{I_{0}}(s) d s \leq 0$ and $a_{3}(t) \geq 0$ for $t \in(0,2 \pi]$, by Lemma 3.2(ii) and (iii), we get

$$
\begin{aligned}
I_{I_{0}}(t) & \leq \sigma_{2}(1+o(1))+\sigma_{1}^{4}(1+o(1)) \int_{0}^{t} a_{3}(s) \cdot\left(\int_{0}^{s} a_{1}(t) \cdot I_{I_{0}}(t) d t\right) d s \\
& =\sigma_{2}(1+o(1))+o(1) \int_{0}^{t} \int_{0}^{s} I_{I_{0}}(t) d t d s
\end{aligned}
$$

and

$$
\begin{aligned}
I_{I_{0}}(t) & \geq \sigma_{1}(1+o(1))+\sigma_{2}^{4}(1+o(1)) \int_{0}^{t} a_{3}(s) \cdot\left(\int_{0}^{s} a_{1}(t) \cdot I_{I_{0}}(t) d t\right) d s \\
& =\sigma_{1}(1+o(1))+o(1) \int_{0}^{t} \int_{0}^{s} I_{I_{0}}(t) d t d s .
\end{aligned}
$$

Hence, for $t \in(0,2 \pi], I_{0} \rightarrow+\infty$, by Lemma 3.2(i) and (3.2)-(3.7), we have

$$
\theta_{I_{0}}(t) \rightarrow 0
$$

and

$$
\sigma_{1}(1+o(1)) \leq I_{I_{0}}\left(t ; \theta_{0}, I_{0}\right) \leq \sigma_{2}(1+o(1)) .
$$


Thus, (i) and (ii) are proved.

To prove (iii), we consider the variational equation of (2.3) about $\theta_{0}$,

$$
\dot{\theta}_{\theta_{0}}=a_{1}(t) \frac{\partial I}{\partial \theta_{0}}+a_{2}(t) \frac{\partial \theta}{\partial \theta_{0}}, \quad \dot{I}_{\theta_{0}}=-a_{2}(t) \frac{\partial I}{\partial \theta_{0}}+a_{3}(t) \frac{\partial \theta}{\partial \theta_{0}} .
$$

Similar to the proof of (ii), one can see that

$$
\sigma_{1}(1+o(1)) \leq \theta_{\theta_{0}}\left(t ; \theta_{0}, I_{0}\right) \leq \sigma_{2}(1+o(1))
$$

for $t \in(0,2 \pi], I_{0} \rightarrow+\infty$. This proves the statement of Lemma 3.3.

Now, we further give the growth estimates on the function $a_{1}(t)$.

Lemma 3.4 Let d satisfy $\left(\mathrm{D}_{3}\right)$.

(i) If $\left|x\left(t ; \theta_{0}, I_{0}\right)\right| \leq d$ for $t \in[0,2 \pi], \theta_{0} \in \mathbb{R}$, and $I_{0} \in \mathbb{R}^{+}$, then there exists a constant $K_{d}>0$, such that $\left|a_{1}(t)\right| \leq \frac{K_{d}}{I^{2}(t)}$.

(ii) If $\left|x\left(t ; \theta_{0}, I_{0}\right)\right| \geq d$ for $t \in[0,2 \pi], \theta_{0} \in \mathbb{R}$, and $I_{0} \in \mathbb{R}^{+}$, then there exists a constant $L_{d}>0$, such that $\left|a_{1}(t)\right| \geq \frac{L_{d}}{I^{2}(t)}$. Moreover, $\left|x\left(t ; \theta_{0}, I_{0}\right)\right| \geq d$ implies that $a_{1}(t)<0$.

Proof (i) If $\left|x\left(t ; \theta_{0}, I_{0}\right)\right| \leq d$ for $t \in[0,2 \pi], \theta_{0} \in \mathbb{R}$, and $I_{0} \in \mathbb{R}^{+}$, we write

$$
M_{d}=\max _{|x| \leq d, t \in[0,2 \pi]}\left|f(t, x)-x f_{x}(t, x)\right| .
$$

Then

$$
\left|a_{1}(t)\right|=\left|\frac{-x\left[f(t, x)-x f_{x}(t, x)\right]}{4 I^{2}}\right| \leq \frac{|x| M_{d}}{4 I^{2}(t)} .
$$

Set $K_{d}=\frac{d M_{d}}{4}$, we have $\left|a_{1}(t)\right| \leq \frac{K_{d}}{I^{2}(t)}$.

(ii) If $\left|x\left(t ; \theta_{0}, I_{0}\right)\right| \geq d$ for $t \in[0,2 \pi], \theta_{0} \in \mathbb{R}$ and $I_{0} \in \mathbb{R}^{+}$, then from condition ( $\left.\mathrm{D}_{3}\right)$, one can see that $x\left[f(t, x)-x f_{x}(t, x)\right]>0, \operatorname{sgn}(x)\left[f(t, x)-x f_{x}(t, x)\right]>b_{\infty}$. Hence, $a_{1}(t)<0$ and

$$
\left|a_{1}(t)\right|=\left|\frac{-x\left[f(t, x)-x f_{x}(t, x)\right]}{4 I^{2}}\right| \geq \frac{|x| b_{\infty}}{4 I^{2}(t)} .
$$

Thus, putting $L_{d}=\frac{d b_{\infty}}{4}$, we have $\left|a_{1}(t)\right| \geq \frac{L_{d}}{I^{2}(t)}$. This completes the proof.

Write $a_{1}(t)=a_{1}^{+}(t)-a_{1}^{-}(t)$, where $a_{1}^{ \pm}(t)=\max \left\{ \pm a_{1}(t), 0\right\}$. To see that the integral of $a_{1}^{+}(t)$ on $[0,2 \pi]$ is smaller than the integral of $a_{1}^{-}(t)$ on $[0,2 \pi]$, we need the following simple lemma.

Lemma 3.5 Let $d$ be as in Theorem 1.1. Define the set $\Delta t=\left\{t \in[0,2 \pi]|| x\left(t ; \theta_{0}, I_{0}\right) \mid \leq d\right\}$. Then there exist $\bar{I}_{0}>0, K_{3}>0$, such that $|\Delta t| \leq \frac{K_{3}}{\sqrt{I_{0}}}$, for all $I_{0} \geq \bar{I}_{0}$.

Proof By Lemma 2.2(i), we have $\Delta t \rightarrow 0$ when and only when $\Delta \theta \rightarrow 0$.

According to the coordinate transformation (2.2), we see that $|\tan \Delta \theta| \leq \frac{d}{\sqrt{2 \gamma I(t)}}$ when $\Delta \theta \rightarrow 0$. Therefore, by using Lemma 2.2(ii), we know that there exist $\bar{I}_{0}>0, K_{3}>0$, such 
that

$$
|\Delta t| \leq \frac{K_{3}}{\sqrt{I_{0}}}
$$

for all $I_{0} \geq \bar{I}_{0}$. Lemma 3.5 follows.

Lemma 3.6 If $I_{0} \gg 1$, then $\int_{0}^{2 \pi} a_{1}(s) d s<0$.

Proof According to the above discussions and Lemma 2.2(ii), we have

$$
\begin{aligned}
\int_{0}^{2 \pi} a_{1}(s) d s & =\left(\int_{a_{1}(s) \leq 0} a_{1}(s) d s+\int_{a_{1}(s) \geq 0} a_{1}(s) d s\right) \\
& \leq-\left(\frac{L_{d}}{\left(k_{2} I_{0}\right)^{2}}(2 \pi-|\Delta t|)-\frac{K_{d}}{\left(k_{1} I_{0}\right)^{2}}|\Delta t|\right) \\
& \leq\left(\frac{\left(k_{2}^{2} K_{d}+k_{1}^{2} L_{d}\right) K_{3}}{\sqrt{I_{0}}\left(k_{1} k_{2} I_{0}\right)^{2}}-\frac{2 \pi L_{d}}{\left(k_{2} I_{0}\right)^{2}}\right) .
\end{aligned}
$$

So, if $\sqrt{I_{0}}>\frac{\left(k_{2}^{2} K_{d}+k_{1}^{2} L_{d}\right) K_{3}}{2 \pi k_{1}^{2} L_{d}}$, then $\int_{0}^{2 \pi} a_{1}(s) d s<0$.

Therefore, in view of Lemma 3.3(ii) and Lemma 3.6, when $I_{0} \gg 1$, the following cannot occur:

$$
\int_{0}^{2 \pi} a_{1}(s) \cdot I_{I_{0}}(s) d s \geq 0
$$

then by (3.2), the case of

$$
\sigma_{1}^{2}(1+o(1)) \int_{0}^{2 \pi} a_{1}(s) \cdot I_{I_{0}}(s) d s \leq \theta_{I_{0}}(2 \pi) \leq \sigma_{2}^{2}(1+o(1)) \int_{0}^{2 \pi} a_{1}(s) \cdot I_{I_{0}}(s) d s
$$

does not happen. Hence, by (3.3), Lemma 3.3(ii), and Lemma 3.6, we have

$$
\begin{aligned}
\theta_{I_{0}}(2 \pi) & \leq \sigma_{1}^{2}(1+o(1)) \int_{0}^{2 \pi} a_{1}(s) \cdot I_{I_{0}}(s) d s \\
& \leq \sigma_{1}^{3}(1+o(1)) \int_{0}^{2 \pi} a_{1}(s) d s<0
\end{aligned}
$$

if $I_{0} \gg 1$.

Using similar arguments to [18], one may extend the Poincaré map $P$ to a new one which is a globally monotone twist map and which is guaranteed by the Aubry-Mather theorem [18]. From the above discussion, we come to the conclusion that there exists $\varepsilon_{0}>0$, such that for any $\alpha \in\left(2 \gamma \pi, 2 \gamma \pi+\varepsilon_{0}\right)$, equation (1.1) has a solution $z_{\alpha}(t)=\left(x_{\alpha}(t), x_{\alpha}^{\prime}(t)\right)$ of AubryMather type with rotation number $\alpha$. Thus, the proof of Theorem 1.1 is completed.

The authors declare that they have no competing interests. 


\section{Author details}

${ }^{1}$ School of Mathematics \& Computer Science, Shangrao Normal University, Shangrao, Jiangxi 334001, China. ${ }^{2}$ Chern Institute of Mathematics, Nankai University, Tianjin, 300071, China. ${ }^{3}$ School of Sciences, Tianjin Chengjian University, Tianjin, 300384, China.

\section{Acknowledgements}

Xiaoming Wang ang Hainv Tan are partially supported by NSFC (No. 11461056). Lixia Wang is partially supported by Tian Yuan Special Foundation (No. 11526148).

Received: 23 February 2016 Accepted: 6 July 2016 Published online: 14 July 2016

\section{References}

1. Aubry, S, Le Daeron, PY: The discrete Frenkel Kontorova model and its extensions: exact results for the ground state. Phys. D, Nonlinear Phenom. 8(13), 384-422 (1983)

2. Mather, JN: Existence of quasiperiodic orbits for twist homeomorphisms of the annulus. Topology 21(4), 457-467 (1982)

3. Bernstein, D, Katok, A: Birkhoff periodic orbits for small perturbations of completely integrable Hamiltonian systems with convex Hamiltonians. Invent. Math. 88(12), 225-241 (1987)

4. Bangert, V: Mather sets for twist maps and geodesics on tori. In: Dynamics Reported, Vol. 1, pp. 1-56 (1988)

5. Moser, J: Recent developments in the theory of Hamiltonian systems. SIAM Rev. 28(4), 459-485 (1986)

6. Moser, J: Monotone twist mappings and the calculus of variations. Ergod. Theory Dyn. Syst. 6(3), 401-413 (1986)

7. Denzler, J: Mather sets for plane Hamiltonian systems. Z. Angew. Math. Phys. 38(6), 791-812 (1987)

8. Casdagli, M: Periodic orbits for dissipative twist maps. Ergod. Theory Dyn. Syst. 7(2), 165-173 (1987)

9. Jiang, MY, Pei, ML: Aubry-Mather theory for twists maps and its applications. Adv. Math. 23(2), 97-114 (1994) (in Chinese)

10. Chow, SN, Pei, ML: Aubry-Mather theorem and quasiperiodic orbits for time dependent reversible systems. Nonlinear Anal., Theory Methods Appl. 25(9), 905-931 (1995)

11. Wang, XM: Quasiperiodic solutions for second order differential equation with superlinear asymmetric nonlinearities and nonlinear damping term. Bound. Value Probl. 2015, 101 (2015)

12. Capietto, A, Liu, B: Quasi-periodic solutions of a forced asymmetric oscillator at resonance. Nonlinear Anal. 56(1), 105-117 (2004)

13. Capietto, A, Dambrosio, W, Liu, B: On the boundedness of solutions to a nonlinear singular oscillator. Z. Angew. Math. Phys. 60(6), 1007-1034 (2009)

14. Wang, XM: Aubry-Mather sets for sublinear asymmetric Duffing equations. Sci. China Math. 42(1), 13-21 (2012) (in Chinese)

15. Wang, XM: Aubry-Mather sets for a class of superlinear asymmetric Duffing equations. Chin. Ann. Math., Ser. A 33(3), 351-358 (2012) (in Chinese)

16. Pei, ML: Mather sets for superlinear Duffing's equations. Sci. China Math. 36(5), 524-537 (1993)

17. Qian, DB: Mather sets for sublinear Duffing equations. Chin. Ann. Math., Ser. B 15(1), 421-434 (1994)

18. Pei, ML: Aubry-Mather sets for finite-twist maps of a cylinder and semilinear Duffing equations. J. Differ. Equ. 113(1), 106-127 (1994)

19. Wang, XM: Aubry-Mather sets for semilinear Duffing equation. Acta Math. Sin. 52(3), 605-610 (2009) (in Chinese)

20. Mather, JN: More Denjoy minimal sets for area preserving diffeomorphisms. Comment. Math. Helv. 60, 508-557 (1985)

21. Wang, YQ: Boundedness for sublinear Duffing equations with time-dependent potentials. J. Differ. Equ. 247(1), 104-118 (2009)

\section{Submit your manuscript to a SpringerOpen ${ }^{\circ}$ journal and benefit from:}

- Convenient online submission

Rigorous peer review

Immediate publication on acceptance

- Open access: articles freely available online

- High visibility within the field

- Retaining the copyright to your article 